Historic, archived document

Do not assume content reflects current scientific knowledge, policies, or practices. 


\section{THE HOME OF}

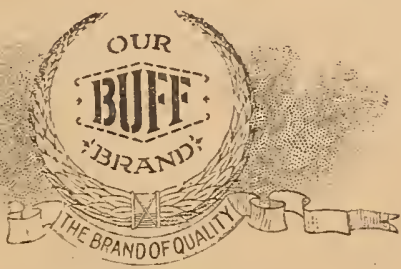

SEEDS

\section{BUFF BRAND SEEDS}

\section{LAWN GRASS AND PASTURE MIXTURES}

\section{QUALITY IS OF GREATEST IMPORTANCE}

OUR LAWN AND PASTURE MIXTURES ARE COMPOSED OF SEEDS OF THE BEST QUALITY AND IN PROPER PROPORTIONS.

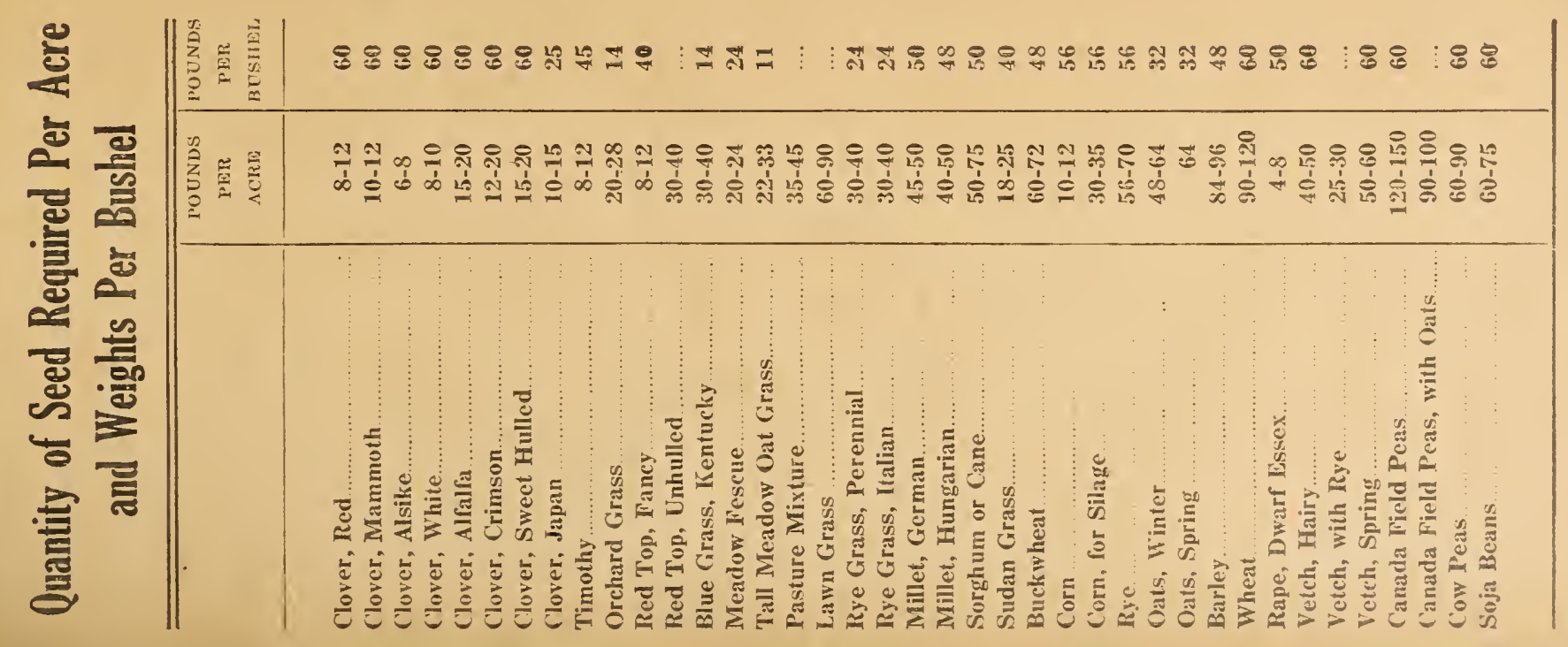




\section{JOHN J. BUFFINGTON \& CO. WHOLESALE SEED MERCHANTS}

\section{8-118 LIGHT STREET}

BALTIMORE, MD., DECEMBER 4, 1926.

\section{RED CLOVER, SAPLING OR MAMMOTH CLOVER, ALSIKE, ALFALFA,} SWEET CLOVER.

MARKET VERY FIRM ON ALL.

MARKET OTHER SEEDS STEADY TO FIRM. ARE BOOKING ORDERS FOR EITHER PROMPT OR LATER SHIPMENT.

WE ARE IN POSITION TO FILL YOUR ORDERS TO GOOD ADVANTAGE.

We do not handle Italian or South European grown Red Clover or Alfalfa.

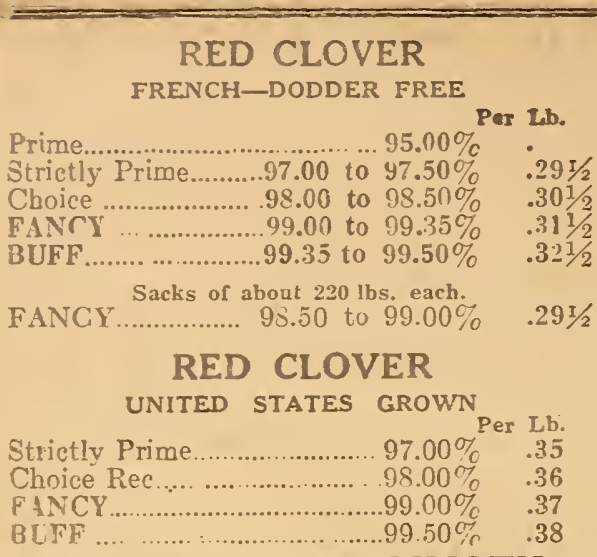

SAPLING OR MAMMOTH

UNITED STATES GROWN Per Li. Choice ............................9.00\% $\quad .38$

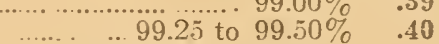
ALSIKE CLOVER

\begin{tabular}{|c|c|}
\hline $\begin{array}{l}\text { Choice. ... } \\
\text { FANCY... } \\
\text { BUFF..... }\end{array}$ & 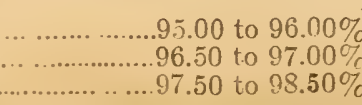 \\
\hline & $\begin{array}{l}\text { ALFALFA } \\
\text { UNITED STATES GROWN }\end{array}$ \\
\hline
\end{tabular}

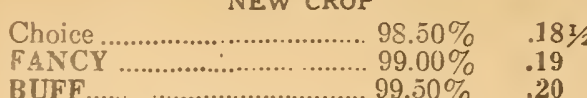

KANSAS ALFALFA BUFF_........... NEW CROP $99.50 \%$ Per

NORTHWESTERN ALFALFA BUFF

CANADIAN VARIEGATED BUFF

GRIMM'S ALFALFA CLOVER BUFF.............ertified........... 99.50\% $\quad .39$ CRIMSON CLOVER FANCY WHITE CLOVER Prime Per Lb

\section{MUMOGWM}

Insures ঐaicker Catch-Bigger Crops CLUVERS

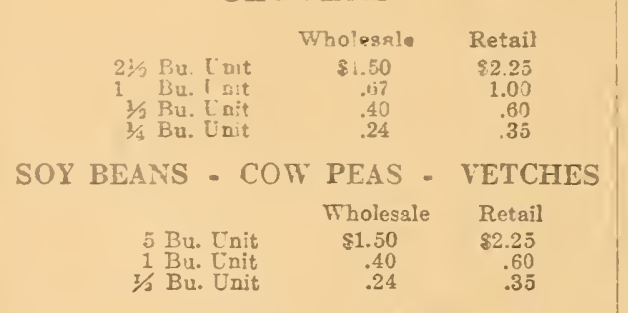

We ouote Firm Prices by wire, when desired, on seeds, car lots or less.

\section{ALASKA PEAS (Sealed) \\ SEED POTATOES (MAINE GROWN)

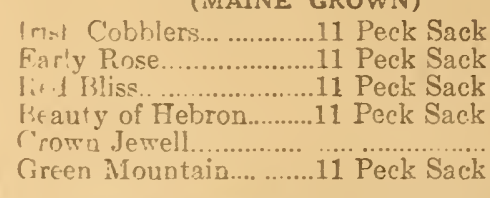

BUFF BRAND POULTRY FEEDS SCRATCHING SIZE

\begin{tabular}{ccc}
\multicolumn{3}{c}{ SWEET CLOVER } \\
SCARIFIED
\end{tabular}

\section{KENTUCKY BLUE GRASS} Choice Kentucky................. $80.00 \% \quad \begin{aligned} & \text { Per Lb. } \\ & .24\end{aligned}$ Fancy Kentucky ..................... $85.00 \% \quad .25$ ORCHARD GRASS NEW CROP $\quad$ Per Bu.

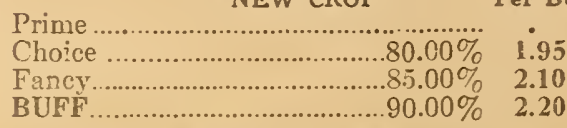

\section{RED TOP/HERD GRASS} Choice Fancy N...........................00\% Per Lb. BUFF Fancy ………………....92.00\% .26 Choice Unhulled.

Finev Unhulled

\section{RYE GRASS}

Perennial...............................98.50\% ${ }^{\text {Per }}{ }^{\text {Lb. }} .13$ $\begin{array}{ll}\text { Italian …….................... } 98.50 \% & .091 / 2 \\ \text { Paceys Short Seeded..............98.50\% } & .141 / 2\end{array}$ CRESTED DOGSTAIL

HARD FESCUE

SHEEP FESCUE

\section{PERMANENT PASTURE}

For Upland ........................ .12 to 14

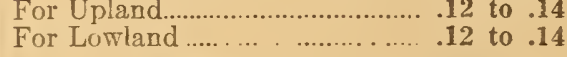
GERMAN MILLET

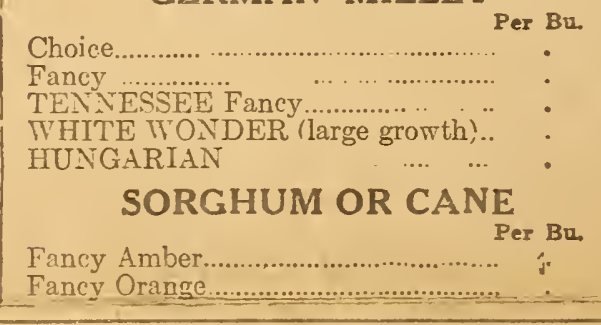

\section{VARIOUS SEEDS Pex Lb. DWARF ESSEX RAPE.................... HAIRY VETCH, FHOICE. \\ HARR VETCH, FANCY \\ JAPAN CLOVER \\ TALL MEADOW OAT GRASS....... $\quad \mathbf{. 1 4}$ \\ MEADOW FESCUE …………........... $\quad .05$
SUDAN GRASS \\ LAWN GRASS \\ BAGS INCLUDED \\ FREIGHT PAID ON LOTS OF 100 LBS. OR MORE Per Lb. Choice .................in bulk..................... .22 Shady Lawn ............................................... $\quad .24$ COW PEAS}

RECLEANED, SACKS FREE. Par BQ. Nixed

Brown Whippoorwill

Gray TVhippoorwill

Blacks................................

New Era

Groit

Brabhan

Gray Crowde..................

Brack Eve, Yirginis SOJA Bears COW PEAS and SOJA BEANS

\section{SOJA BEANS}

RECLEANED, SACKS FREE Per Ba. Mammoth Yellow................................. Manchu

Wilson............

Virginia..............

Hollybrook

Tar Heel

\section{CANADA FIELD PEAS}

Choice AMERICAN GROWN Per Bu. Fancy

\section{SEED GRAIN} BAGS EXTRA

Maryland Winter seed Rye . Per Ba.

Rosen Seed Rye

Abruzzi Seed Rye.

Buckwheat. Japanese...

FULTZ WHEAT.

Leafs Prolific Wheat

China Wheat

BARLEY

Winter Seed Oats (Virginia Gray)

Burt Oats

ENSILAGE CORN (Southern White)

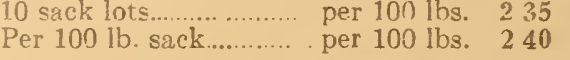
CHICK SIZE 10 sack lots...................per 100 lbs. 250 per $100 \mathrm{lbs} .255$

\section{MISCELLANEOUS FEEDS}

DRY MASE $\quad \ldots . . .$. per $10 n$ lhs. 2.50 Beef Scraps..............50\% per $100 \mathrm{lbs} 4 \mathrm{n} 0$ Crushed Bone.................per 100 lbs. 3.00 Meat Meal .............50\% per 1mo the. 4.00 Digester Tankage..60\% per 100 Tbs. 3.50 Kaffir rorn.......................per 100 lbs Sunforer..................................... per lb. $\quad .05$ Hemp................................ per lb. Buckwheat ............................. per lb. .023 Granalated Chareoal...... per $1^{n} 0^{\prime}$ 'bs. 2.30 Corada Field Peas ............ per lh. per th.
Millet Alfalfa Meal ................ 100 lbs 2.20 CRUSHED OYSTER SHELL. Ton lots ... Per $100 \mathrm{lb}$. sack 